Gynäkologe 2009 • 42:327

DOI 10.1007/s00129-009-2357-3

Online publiziert: 24. April 2009

๑) Springer Medizin Verlag 2009

\author{
H.G. Bender ${ }^{1} \cdot$ K. Vetter ${ }^{2}$ \\ ${ }^{1}$ Universitäts-Frauenklinik, Heinrich-Heine-Universität, Düsseldorf \\ ${ }^{2}$ Vivantes-Klinikum Neukölln, Berlin
}

\title{
40 Jahre Kardiotokographie
}

W. Künzel stellt die Entwicklungsarbeiten für die technische Konfiguration und die ersten Anwendungserprobungen und -auswertungen dar. Nach der technischen Etablierung der Methode haben sich mehrere Arbeitsgruppen und Einzeluntersucher um eine korrekte Interpretation der Kardiogramme, z. B. in Form von Scores, und die Vermeidung von Fehlern in der anfangs nicht einfachen Auswertung der Aufzeichnungen bemüht.

\section{> Jahrzehntelang wurde die sachgerechte Deutung von CTG-Daten diskutiert}

Die CTG-Entwicklung war auch in den USA frühzeitig aufgegriffen worden und breitete sich in anderen Ländern ebenfalls verhältnismäßig rasch aus. Dadurch ergaben sich jahre- und jahrzehntelange wissenschaftliche Dispute um die richtige Anwendung und die sachgerechte Deutung, die durch die zunehmende Erfahrung einigem Wandel unterworfen war. Doumouchtsis und Arulkumaran stellen ihren aktiven Beitrag zu diesem Prozess dar.

Eine jahrelange erbitterte Auseinandersetzung betraf die Frage, ob das CTG oder die MBU die aussagekräftigere Methode für die Überwachung des Fetus und damit als überlegen anzusehen sei. Dieses Thema beherrschte viele Kongresse der damaligen Zeit und wurde sicher auch durch Schulen-"Überzeugungen" und Emotionen angeheizt. K. Vetter, Nachfolger von Saling, beleuchtet die Entwicklung in der Anwendung der beiden Methoden und die heutige ergänzende Positionierung.

Nach 40 Jahren CTG-Anwendung drängt sich die Frage auf, ob wir inzwischen an einem Punkt angelangt sind, an dem die Methode als fest etabliert angese- hen werden kann, aber keine weiteren Entwicklungsmöglichkeiten unter klinischen und technischen Gesichtspunkten mehr bietet. Nonnenmacher, Hopp und Dudenhausen schildern ihre Bewertung der zukünftigen Erweiterung und Präzisierung des Einsatzes in der geburtshilflichen Praxis.

M. Späth schildert aus dem Blickwinkel der Industrie die Herausforderung, technische Innovationen sowohl für die Ansprüche in den Industrie- als auch in den Schwellenländern zu entwickeln und erfolgreich zum Einsatz zu bringen.

Das 40-jährige Jubiläum der CTGTechnik war Ausgangspunkt für diese Übersicht. Die Herausgeber hoffen, nicht nur Einblicke in die historische Entwicklung dieser Methode gegeben zu haben, sondern auch zusätzliche Ansatzpunkte für eine optimale, auf wissenschaftlichen Erkenntnissen basierende Anwendung und Interpretation.



H. G. Bender

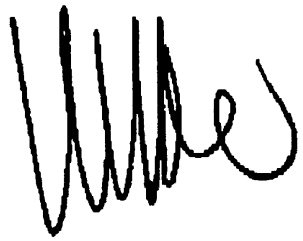

K. Vetter

\section{Korrespondenzadresse}

Prof. Dr. H.G. Bender

Universitäts-Frauenklinik, Heinrich-Heine-Universität Moorenstraße 5, 40225 Düsseldorf benderhg@uni-duesseldorf.de 